For Publisher's use

\title{
NEW RESULTS FOR HEAVY FLAVOR AND QCD TESTS AT THE TEVATRON
}

\author{
DONATELLA LUCCHESI \\ FOR THE CDF AND D0 COLLABORATIONS \\ Dipartimento di Fisica Galileo Galilei, \\ Via Marzolo 8, 35131 Padova \\ Italyemail:Donatella.Lucchesi@pd.infn.it
}

\begin{abstract}
The latest results for QCD and Heavy flavor at the Tevatron are summarized. The most recent results on Jet physics are discussed in the light of searching for new physics and to study the contribution of soft interactions to the hard process. The production of $c$ and $b$-hadrons and the their decay properties can be precisely measured at the Tevatron. Lifetimes, branching fractions and CP asymmetries for several decay modes are discussed. In particular the prospects for $B_{s}$ mixing at the Tevatron are presented.
\end{abstract}

\section{The accelerator complex and the experiments}

In this decade both experiments CDF and D0 aim to perform high precision measurements in the Heavy Flavor and Quantum Chromodynamics (QCD) physics sector investigating process with a relative high production cross sections. New phenomena, with a cross sections several order of magnitude lower than inelastic $p \bar{p}$ cross section are also searched for. Since these process compete among them for the different production and decay properties the accelerator complex and the detectors have been upgraded to cope with all the requirements.

\subsection{The new machine}

In order to obtain large samples of data two steps have been taken: increase the centerof-mass energy and increase the luminosity. The first one has been raised from 1.8 to $1.96 \mathrm{TeV}$. In order to increase the instantaneous luminosity the accelerator complex has been changed to allow 36x36 bunches per beam. The highest peak luminosity up today has been $1.1032 \times 10^{32} \mathrm{~cm}^{-2} \mathrm{~s}^{-1}$. The inter bunches time has been lowered to $396 \mathrm{~ns}$ from 3500 ns to keep the average number of interactions per crossing acceptable. The machine is operating now around 120 hours per week with stable performances. The delivered integrated luminosity is now $0.7 \mathrm{fb}^{-1}$ as from design. Starting from these numbers the expected integrated luminosity in the 2005 goes from 0.93 to $1.2 \mathrm{fb}^{-1}$ depending on the machine performances. At the end of 2009 the Tevatron should deliver $4.4-8.5 \mathrm{fb}^{-1}$ of data.

\subsection{CDF and DO experiments}

CDF and D0 are multi-purpose detectors giving the possibility to perform measurements in different physics fields. An high resolution tracking system is the heart of the detectors and it is crucial for $B$-physics. The CDF tracker is based on silicon microstrips detectors and drift chamber. D0 uses silicon microstrips, pixels and scintillating fiber detector. Both experiments have upgraded the tracking system to cover high $\eta$ regions which are important also for muons identification in the very forward region. For $B$-physics are also important Particle Identification Detectors (PID). CDF uses the measurement of the time of flight of each particle to distinguish $\pi$ from $K$ and $p$ and the energy deposited on the drift chamber. The achieved separation $\pi-K$ is about $1.5 \sigma$ up to $5 \mathrm{GeV}$. Good electromagnetic calorimeter and well calibrated hadron calorimeter covering as much solid an- 


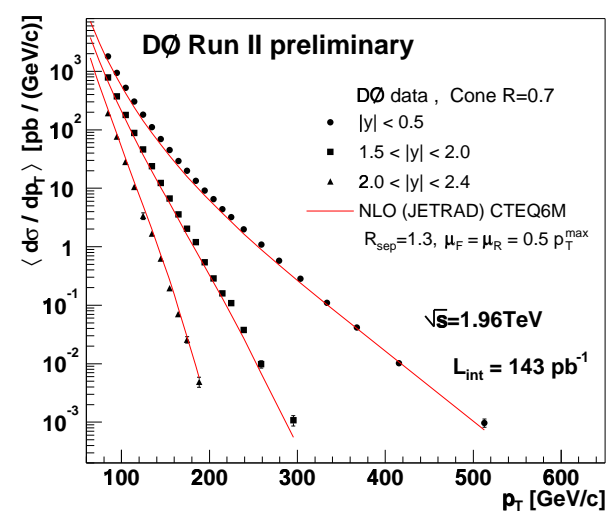

Figure 1. D0: Inclusive jet cross section in different regions of rapidity compared to $\mathrm{pQCD}$ NLO predictions.

gle as possible are necessary for good jet energy resolution. In addition to the detector changes, the trigger and the data acquisition systems have been upgraded to cope with the higher collision rates. In particular CDF has a new trigger, SVT, which allows to select events with at least two tracks displaced from the primary vertex. Large samples of $c$ and $b$ hadrons are collected with this trigger.

\section{Jet Physics at $2 \mathrm{TeV}$}

Jets have been studied at the Tevatron as means of searching for new particles and interactions, testing a variety of perturbative QCD (pQCD) predictions and providing input for the global Parton Distribution Functions (PDF) fits. The increased instantaneous luminosity and center-of-mass energy with the improved detectors will allow stringent tests of the Standard Model (SM) predictions in extended regions of jet transverse momentum, $P_{T}^{j e t}$, and jet rapidity, $Y^{j e t}$.

\subsection{Inclusive Jet production}

In the so called Run I there was a great interest in the measurement of the inclusive jet

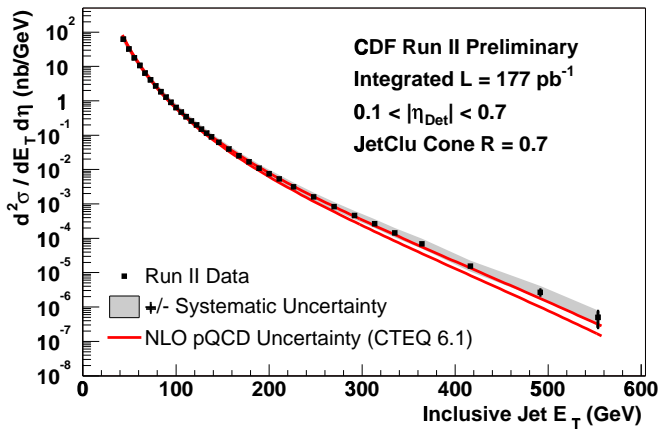

Figure 2. CDF: measured inclusive jet cross section compared to $\mathrm{pQCD}$ NLO prediction.

cross section due to the excess appeared at high jet transverse energy, $E_{T}$, over the Nextto-Leading Order (NLO) theoretical predictions which were using the available PDF's. The inclusion of the CDF and D0 measurements in the global fits explained this excess in term of a larger than expected gluon distribution at high $x$. The uncertainties on the gluon PDF at high $x$ are still large and they can be better constrained measuring the inclusive cross section in the forward regions. D0 experiment has already performed a such a measurement with $\left|Y^{\text {jet }}\right|<2.4$ (figure 1). At the moment the results are affected by a large systematic error dominated by the jet energy scale. The experiment is highly reducing this error and a much more precise measurement will allow to constrain the gluon distribution enhancing the sensitivity for new physics in the jet cross section. Thanks to the increased center-of-mass energy the measurement of the inclusive jet cross section has already extended the $E_{T}$ coverage. CDF measurement goes up by almost $150 \mathrm{GeV}$ compared to Run I as showed in figure 2 allowing to test new regions for new physics. If new phenomena are presents they should manifest them self also in the di-jets cross section as an excess in the high mass di-jets invariant mass distribution or as resonances in this 


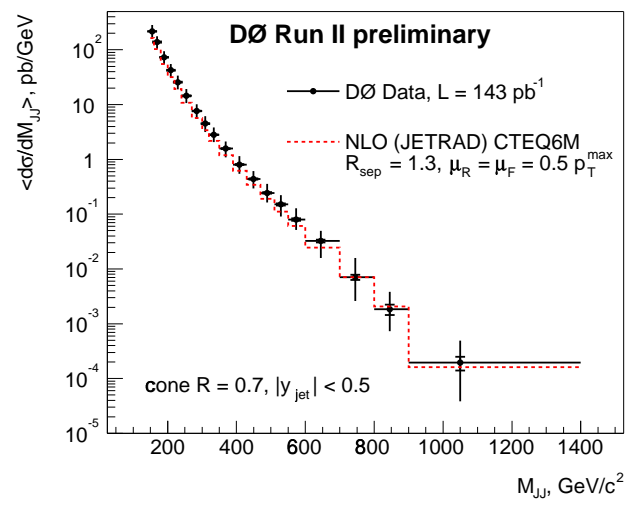

Figure 3. D0: jet cross section as function of di-jets invariant mass compared to pQCD NLO prediction.

mass region. The comparison between data and theory, pQCD NLO, (figure 3) shows an agreement within the large experimental error dominated by the systematic on the jet energy scale. The measurement of the inclusive jet cross section at low $P_{T}^{\text {jet }}$ allows to study the jet fragmentation. CDF determines the inclusive jet cross section using the $K_{T}$ algorithm with the value of the $D$ parameter in the $K_{T}$ expression,

$K_{i j}=\min \left(p_{T, i}^{2}, p_{T, j}^{2}\right) \frac{\left(y_{i}-y_{j}\right)^{2}+\left(\phi_{i}-\phi_{j}\right)^{2}}{D}$ equal to $0.5,0.7$ and 1.0. The comparison between data and pQCD NLO shows that data is systematically above the predictions at low $P_{T}^{\text {jet }}$ and that the effect increases as $D$ increases (see figure 4 ). This behavior can be explained as the missing soft-gluon contributions and a not complete fragmentation description. More details on these measurements can be found here ${ }^{1}$.

\section{2 $W+j e t(s)$ Production}

The study of the associated production of a $W$ boson and a given number of jets is important for the precise determination of the Top quark properties and to evaluate the characteristics of the background in the Higgs

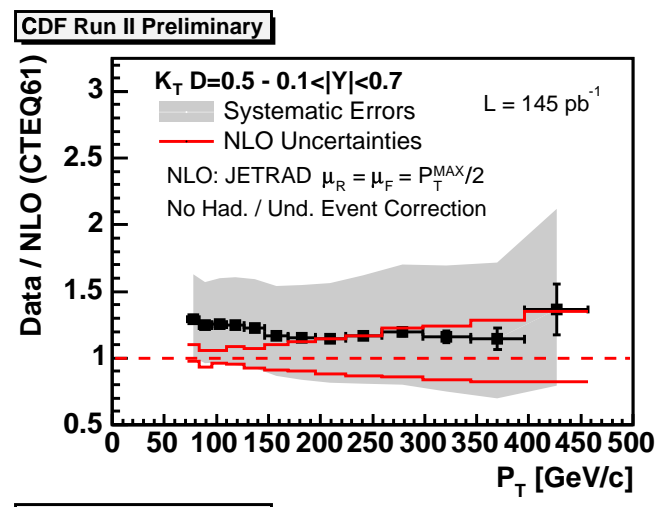

CDF Run II Preliminary

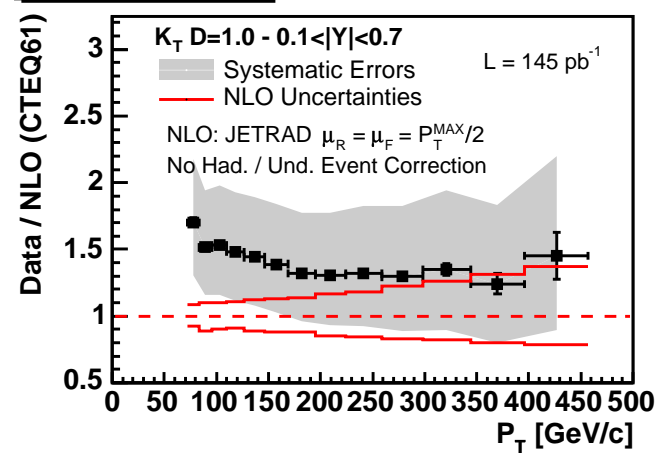

Figure 4. CDF: Ratio between the inclusive jet cross section evaluated using the $K_{T}$ algorithm with $D=$ 0.5 (up) and $D=1.0$ (bottom) and pQCD NLO predictions. 


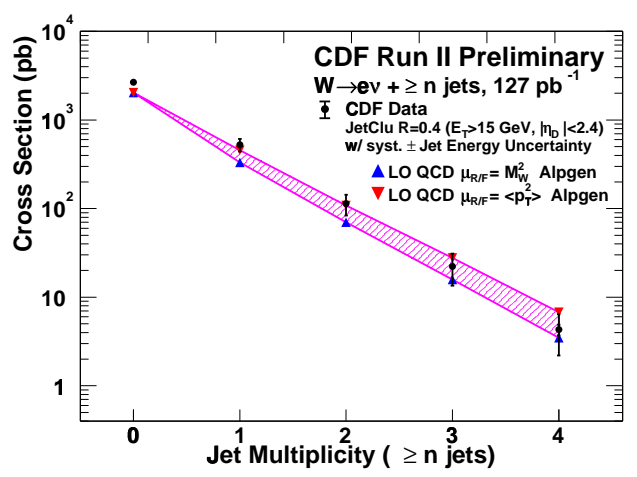

Figure 5. CDF: Measured inclusive cross section for $W+\geq N_{\text {jet }}$ compared to pQCD LO prediction as implemented in ALPGEN+HERWIG

searches. The precise measurement of the $W+N_{\text {jets }}$ cross section is one of the means to test the $\mathrm{pQCD} \mathrm{LO}$ and NLO. Figure 5 shows the CDF cross sections compared with the leading-order QCD prediction from the matrix-element Alpgen Monte Carlo program interfaced with the parton-shower model provided by the Herwig Monte Carlo program. The Run II measurement is about $10 \%$ larger than in Run I as expected for the increased center-of-mass energy.

\section{Heavy Flavor Production}

The $b$ and $c$ quark production cross section should be well calculable in QCD since $m_{Q}>>\Lambda_{Q C D}$ (where $Q=b, c$ ). The Beauty quark production cross section has been measured by both the CDF and D0 experiments in Run I and found to be about 3 times larger than NLO QCD calculation. Since then several theoretical improvements took place: the use of the Fixed Order Next to Leading Log calculations, the inclusion of the new fragmentations functions extracted by LEP data, the use of new PDF's and of the Monte Carlo which match the NLO calculation with parton shower formalism in Herwig have almost canceled the discrepancy. Never-

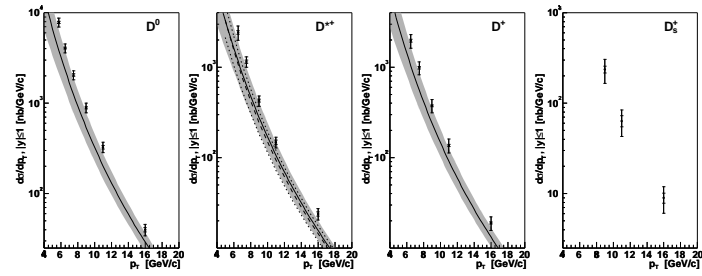

Figure 6. CDF: Differential cross section for $|y|<1$ shown by the points. The solid curves are the theoretical predictions with the uncertainties indicated by the shaded bands.

theless, new measurements at the new centerof-mass energy are important to shed light on this controversial field. Using just $5.8 \pm 0.3$ $\mathrm{pb}^{-1}$ data CDF performs a measurement of prompt charm meson production cross section. The charm mesons are reconstructed in the following decay modes: $D^{0} \rightarrow K^{-} \pi^{+}$, $D^{*+} \rightarrow D^{0} \pi^{+}$with $D^{0} \rightarrow K^{-} \pi^{+}, D^{+} \rightarrow$ $K^{-} \pi^{+} \pi^{+}, D_{s} \rightarrow \phi \pi^{+}$with $\phi \rightarrow K^{+} K^{-}$and their charge conjugate.

The measured differential cross sections are compared to a recent calculations ${ }^{2}$ as shown in figure 6 . They are higher than the theoretical predictions but compatible within uncertainties.

A new measurement of the total inclusive $J / \psi$ cross section in the central rapidity region $|y|<0.6$ has been carried out and the integrated value is:

$$
\begin{aligned}
& \sigma(p \bar{p} \rightarrow J / \psi X) \times B r(J / \psi \rightarrow \mu \mu)= \\
& 240 \pm(\text { stat })_{-28}^{+35}(\text { syst }) n b
\end{aligned}
$$

Taking advantage of the large azimuth coverage of its muon system, D0 measured the differential cross section as function of the rapidity. Both are in agreement with the theoretical predictions.

Selecting $b$ hadrons, $H_{b}$, with $H_{b} \rightarrow$ $J / \psi+X$ CDF has measured also the $H_{b}$ cross section shown in figure 7 with the Run I measurements and the new theoretical predictions superimposed where is possible to see the good agreement between Run II and Run I data and data and theory.

The $b$-jet production cross section anal- 


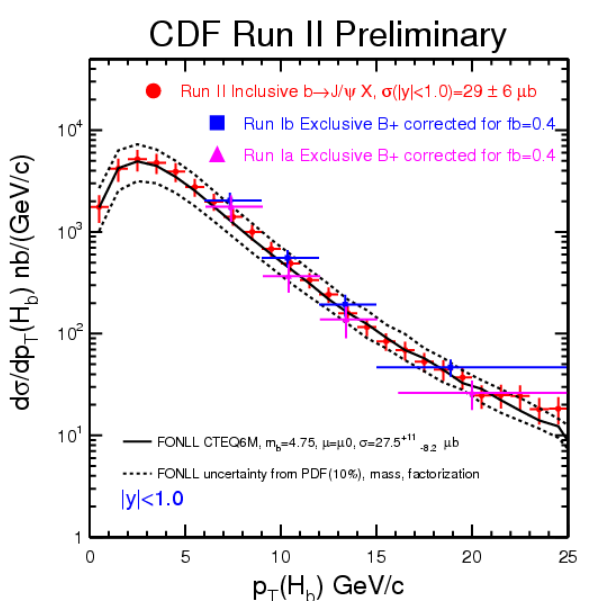

Figure 7. CDF: Differential $b$ hadron cross section as function of $b$ hadron momentum compared to theory predictions.

ysis is in progress for CDF and D0 measured it on $3.4 \mathrm{pb}^{-1}$ of data. The $b$ is identified thought the semileptonic decay to muon. The result, which will be updated soon, is consistent with Run I measurement. Details on all cross section measurements can be found here ${ }^{3}$.

\section{$4 \quad B$ Hadron Lifetimes and $B_{s}^{0}$ Lifetime Difference}

At the first order the $b$-hadron lifetimes are expected to be:

$$
\tau\left(B_{u}^{+}\right) \approx \tau\left(B_{d}^{0}\right) \approx \tau\left(B_{s}^{0}\right) \approx \tau\left(\Lambda_{b}^{0}\right)
$$

Spectator effects can be calculated in the Heavy Quark Expansion Theory (HQET) ${ }^{4}$ which modify the lifetime ratios:

$$
\begin{aligned}
& \frac{\tau\left(B_{u}^{+}\right)}{\tau\left(B_{d}^{0}\right)}=1.09 \pm 0.03, \\
& \frac{\tau\left(B_{s}^{0}\right)}{\tau\left(B_{d}^{0}\right)}=1.00 \pm 0.01, \\
& \frac{\tau\left(\Lambda_{b}^{0}\right)}{\tau\left(B_{d}^{0}\right)}=0.87 \pm 0.05
\end{aligned}
$$

Experimentally the Tevatron experiments can not compete with the $B$-factories for the $B_{u}^{+}$and $B_{d}^{0}$ measurements while they are the unique place where $B_{s}^{0}$ and $\Lambda_{b}^{0}$ lifetimes can be precisely measured. Anyhow both experiments CDF and D0 measure the $B_{u}^{+} / B_{d}^{0}$ lifetime ratio as a crosscheck that the technique and all the detector effects are well under control. The value obtained by $\mathrm{CDF}$ is $\tau\left(B_{u}^{+}\right) / \tau\left(B_{d}^{0}\right)=1.080 \pm 0.042$ where the $B_{d}^{0}$ is reconstructed via the decay in $J / \psi K^{* 0}$ and $B_{u}^{+} \rightarrow J / \psi K^{+}$. D0 reconstructs $B \rightarrow$ $\mu^{+} \nu D^{*-} X$ which is constituted for $86 \%$ of $B^{0}$ and $B \rightarrow \mu^{+} \nu D^{0} X$ populated by $82 \%$ of $B^{+}$. Performing a ratio bin by bin of the proper decay length obtains $\tau\left(B_{u}^{+}\right) / \tau\left(B_{d}^{0}\right)=$ $1.093 \pm 0.021$ (stat) \pm 0.022 (syst). Both measurements are in agreement with the world average $\tau\left(B_{u}^{+}\right) / \tau\left(B_{d}^{0}\right)=1.086 \pm 0.017$.

In $250 \mathrm{pb}^{-1}$ of data $\mathrm{D} 0$ reconstructs $61 \pm$ 12 events of $\Lambda_{b}^{0} \rightarrow J / \psi \Lambda^{0}$ and $291 \pm 23$ events of $B_{d}^{0} \rightarrow J / \psi K_{s}^{0}$. The measured lifetime ratio $\tau\left(\Lambda_{b}^{0}\right) \tau\left(B_{d}^{0}\right)=0.874_{-142}^{+0.169}($ stat $) \pm 0.028$ (syst) is in good agreement with the theoretical expectations.

The lifetime measurements in the $B_{s}^{0}$ sector depend on the decay channel used due to the fact that the sample can have different amount of the two component $B_{L}$ and $B_{H}$, the mass eigenstates with different mass and width corresponding also to different $\mathrm{CP}$ states. The theoretical expectations for $\Delta \Gamma=\Gamma_{L}-\Gamma_{H}$ range from $(7-14) \times 10^{-2}$. CDF exploits the decay $B_{s}^{0} \rightarrow J / \psi \phi$ where the final state is composed by two spin-1 particles and can be decomposed through an angular analysis into CP-odd and CP-even states. The three complex amplitude, the short-lived CP-even $A_{0}$ and $A_{\|}$, and the longlived CP-odd $A_{T}$ are extracted from a fit to the data:

$$
\begin{aligned}
& A_{0}=0.784 \pm 0.039 \pm 0.007 \\
& \left|A_{\|}\right|=0.510 \pm 0.082 \pm 0.013 \\
& \left|A_{T}\right|=0.354 \pm 0.098 \pm 0.003 \\
& \arg \left(A_{||}\right)=1.94 \pm 0.36 \pm 0003
\end{aligned}
$$

The same fit gives a lifetime difference $\Delta \Gamma=$ $0.47_{-0.24}^{+0.19} \pm 0.01 \mathrm{ps}^{-1}$ and $\Delta \Gamma / \Gamma=0.65_{-0.33}^{+0.25} \pm$ 0.01 when no constrain is applied on total $B_{s}$ width. If $\Gamma_{s}=\Gamma_{d}$ is required, $\Delta \Gamma=$ $0.46_{-0.18}^{+0.17} \pm 0.01 \mathrm{ps}^{-1}$ and $\Delta \Gamma / \Gamma=0.71_{-0.28}^{+0.24} \pm$ 0.01 . In figure 8 the projection of the unbinned maximum likelihood fit on $c t$ is shown. 


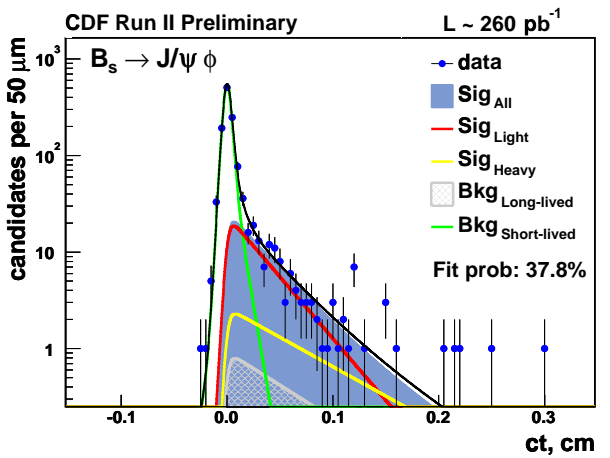

Figure 8. CDF: Projection of unbinned maximum Likelihood fit.

For a complete review of lifetime measurements see ${ }^{5}$.

\section{Branching Ratios and CP Asymmetries}

CDF has performed several measurements exploiting the displaced tracks trigger, SVT. First of all a new decay mode $B_{s}^{0} \rightarrow \phi \phi$ with $\phi \rightarrow K K$ has been reconstructed and the branching ratio measured to be:

$$
\begin{gathered}
B R\left(B_{s}^{0} \rightarrow \phi \phi\right)=(1.4 \pm 0.6(\text { stat } .) \pm \\
\left.0.2(\text { syst. }) \pm 0.5\left(B R^{\prime} s\right)\right) \times 10^{-5}
\end{gathered}
$$

This decay mode can be used to measure the $B_{s}^{0}$ lifetime difference and test the Standard Model for new physics. Details on this measurement can be found here ${ }^{6}$.

With the same trigger a sample of $B \rightarrow$ $h^{+} h^{\prime}-(\pi, K)$ has been recorded. The mass peaks of all these two-body decays are reconstructed with a conventional assignment of pion mass to both prongs, and contribute to a single bump see figure 9 . In the measurement of the relative Branching Ratios and the direct $\mathrm{CP}$ asymmetry the small kinematics differences between distinct modes and the particle identification information provided by the specific ionization $(d E / d x)$ in the drift chamber are exploited. The results on Branching Ratios are:

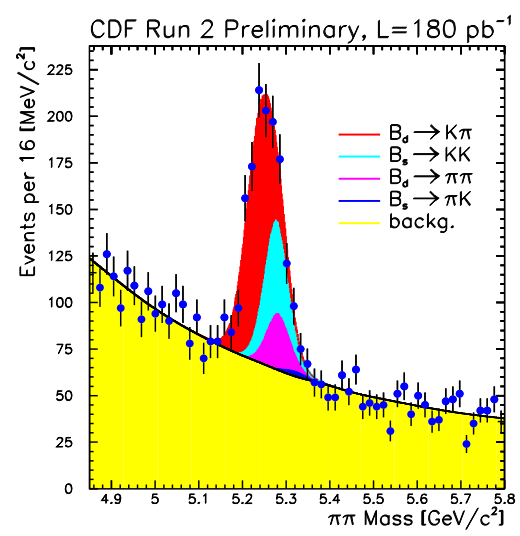

Figure 9. CDF: Two track invariant mass distribution assuming the $\pi$ mass.

$$
\begin{aligned}
& \frac{B R\left(B_{d}^{0} \rightarrow \pi^{+} \pi^{-}\right)}{B R\left(B_{d}^{0} \rightarrow \pi^{+} K^{-}\right)}=0.24 \pm 0.06(\text { stat }) \pm 0.05(\text { syst }) \\
& \frac{f_{s}}{f_{d}} \frac{B R\left(B_{s}^{0} \rightarrow K^{+} K-\right)}{B R\left(B_{d}^{0} \rightarrow \pi^{+} K^{-}\right)}=0.50 \pm 0.08(\text { stat }) \pm 0.07(\text { syst }) \\
& \frac{B R\left(B_{s}^{0} \rightarrow \pi^{+} \pi^{-}\right)}{B R\left(B_{s}^{0} \rightarrow K^{+} K^{-}\right)}<10 @ 90 \% C . L . \\
& \frac{f_{s}}{f_{d}} \frac{B R\left(B_{s}^{0} \rightarrow K^{+} \pi-\right)}{B R\left(B_{d}^{0} \rightarrow \pi^{+} K^{-}\right)}<0.11 @ 90 \% C . L
\end{aligned}
$$

The direct $\mathrm{CP}$ asymmetry is given:

$$
\begin{array}{r}
A_{\mathrm{CP}}=\frac{N\left(\bar{B}_{d}^{0} \rightarrow K^{-} \pi^{+}\right)-N\left(B_{d}^{0} \rightarrow K^{+} \pi^{-}\right)}{N\left(\bar{B}_{d}^{0} \rightarrow K^{-} \pi^{+}\right)+N\left(B_{d}^{0} \rightarrow K^{+} \pi^{-}\right)}= \\
-0.04 \pm 0.08(\text { stat. }) \pm 0.01(\text { syst. })
\end{array}
$$

Details on this work can be found here ${ }^{7}$.

Branching ratios and $\mathrm{CP}$ asymmetries are measured by CDF also in the charm sector for $D^{0} \rightarrow K K$ and $D^{0} \rightarrow \pi \pi$. CDF alone improves the current world averages. A complete description of this measurements can be found here ${ }^{8}$

\section{$6 \quad B_{c}$ and $B^{* *}$ evidences}

The $B_{c}$ is the last of the ground state $B$ meson to be studied. Many phenomenological models $\left(\begin{array}{lll}9 & 10 & 11\end{array}\right)$ predict lifetime, mass and branching fractions to a rich array of final states. The properties of the $B_{c}$ measured in Run I had the limitation of the low statistics. The $B_{c}$ decays a significant fraction of the times to final states containing a 

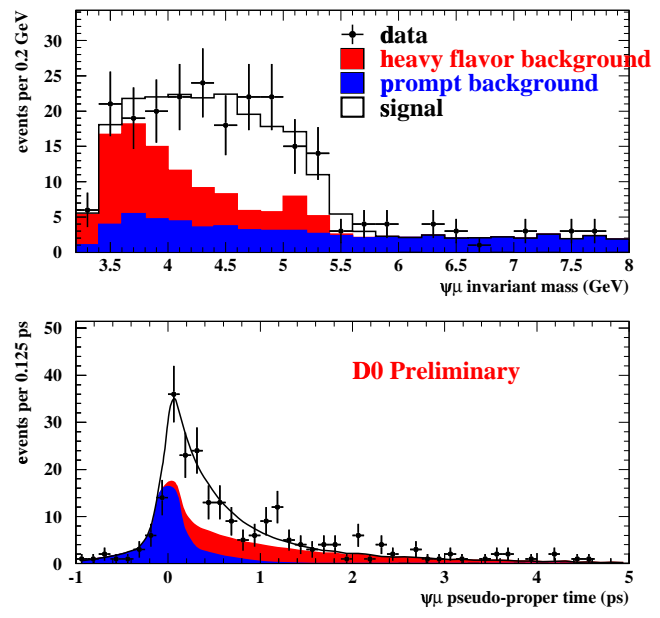

Figure 10. D0: The $J / \psi \mu$ invariant mass and pseudoproper time distributions for data(points) with the fit result overlaid.

$J / \psi$ which is an clear signature at the Tevatron. D0 has identified $B_{c}$ candidates in the $B_{c} \rightarrow J / \psi \mu X$ final state. An unbinned likelihood fit of the mass and the lifetime is performed for each candidate. The probability distribution of the pseudo-proper time is obtained from an exponential distribution taking into account the resolution on the eventby-event basis and correcting for the loss of the neutral particles. The fit is performed under a variety of of mass hypotheses. A parabolic fit to the values of $-2 \log$ (likelihood) returned by the fit yields a mass estimate of $5.95_{-0.13}^{+0.14} \mathrm{GeV} / \mathrm{c}^{2}$, a lifetime of $0.448_{-0.096}^{+0.123}$ ps and the number of events $N=95 \pm 12$. The results of the best fit are shown in figure 10. The major systematic errors are coming from the limited background sample, the non-resonant $B_{c}$ decays, the tracking detector alignment and the transverse momentum binning. The final results are: $\mathrm{N}=95 \pm 12 \pm 11$ lifetime $0.448_{-0.096}^{+123} \pm 0.121$ ps and mass $5.95_{-0.13}^{0.14} \pm 0.34 \mathrm{GeV} / \mathrm{c}^{2} 12$.

Besides new ground states also the spectroscopy of mesons containing b-quark can

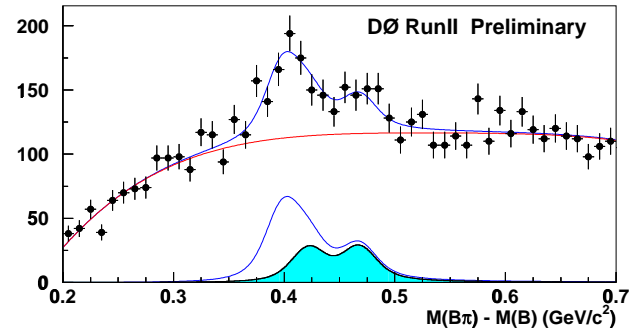

Figure 11. D0: Mass difference $\Delta M=M(B \pi)-$ $M(B)$. The fit result is overlaid with the background contribution. The lower lines show the signal with the background subtracted. The filled histogram shows the contribution of the $B_{2}$

be extensively studied at the Tevatron. Only the $J^{p}=1^{-}$excited state $B^{*}$ is considered well established. The narrow $L=1 B_{1}$ and $B_{2}$ have been studied by ALEPH ${ }^{13}$ with exclusive $B$ decays but they were statistically limited. On the contrary the mass, widths and decay branching fractions of these states are predicted with good precision by various theoretical models $\left({ }^{14}{ }_{-}{ }^{17}\right)$. These predictions can be verified experimentally to obtain important information on the quark interaction inside bound states and derive constrains on non-perturbative QCD. The D0 collaboration studied the properties of the two narrow states with $L=1$. The decay chain is: $B_{1} \rightarrow B^{*} \pi, B^{*} \rightarrow B \gamma$ and $B_{2} \rightarrow B^{*} \pi$ with $B^{*} \rightarrow B \gamma$ plus the direct decay $B_{2} \rightarrow B \pi$. The $B$ are reconstructed in the following final states: $B^{+} \rightarrow J / \psi K^{+}, B_{d}^{0} \rightarrow J / \psi K^{* 0}$ and $B_{d}^{0} \rightarrow J / \psi K_{s}$. An additional track $(\pi)$ with the right charge correlation is required for $B_{1}$ while no charge requirements are applied to $B_{2}$. Figure 11 shows the mass difference $\Delta M=M(B \pi)-M(B)$. The contribution of $B_{1} \rightarrow B^{*} \pi$ is evident in the first peak. The second one is the contribution of $B_{2} \rightarrow B^{*} \pi$ and $B_{2} \rightarrow B \pi$. The number of the events is extracted from the fit to be $536 \pm 114$ for both decay channels. The results for the 


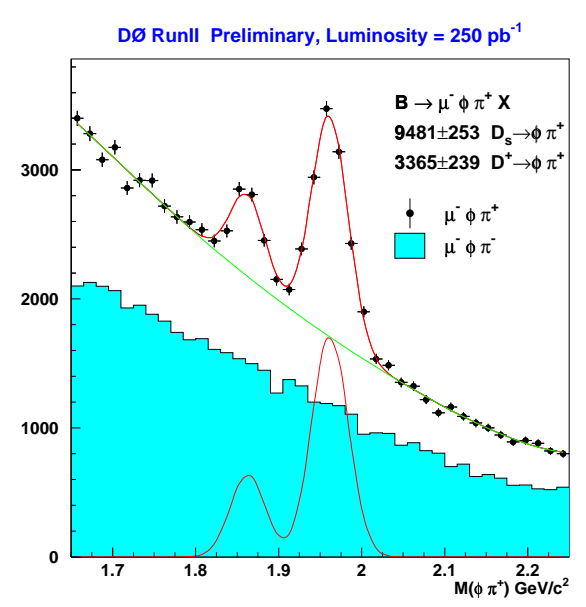

Figure 12. Semileptonic $B_{s}$ yields for D0

masses and the widths including systematic errors are:

$$
\begin{aligned}
& M\left(B_{1}\right)=5724 \pm 4(\text { stat }) \pm 7(\text { syst }) M e V / c^{2} \\
& M\left(B_{2}\right)-M\left(B_{1}\right)= \\
& 23.6 \pm 7.7(\text { stat }) \pm 3.9(\text { syst }) M e V / c^{2} \\
& \Gamma_{1}=\Gamma_{2}=23 \pm 12(\text { stat }) \pm 9(\text { syst }) M e V / c^{2}
\end{aligned}
$$

They are in agreement with the theoretical expectations within the errors.

\section{$7 \quad B$ Mixing}

The measurement of the $B_{s}^{0}$ oscillation frequency, $\Delta m_{s}$, is one of the missing pieces to constrain the Unitarity Triangle and therefore the Standard Model. This model gives the value of $\Delta m_{s}$ with an accurate prediction and the comparison with the experimental measurements allows to test the presence of new physics beyond the Standard Model. Moreover the knowledge of $\Delta m_{s}$ is a prerequisite for many time-dependent $\mathrm{CP}$ violation studies in the $B_{s}$ system. The current limit is $\Delta m_{s}>14.4 p s^{-1}$. The value for the $B_{d}^{0}$ mixing frequency $\Delta m_{d}=0.502 \pm 0.007$. The comparison between the two frequencies tell us how difficult will be to extract $\Delta m_{s}$ from data, there will be more than three full $B_{s}^{0}$ oscillation cycles within one lifetime. In order to perform the measurement the following is

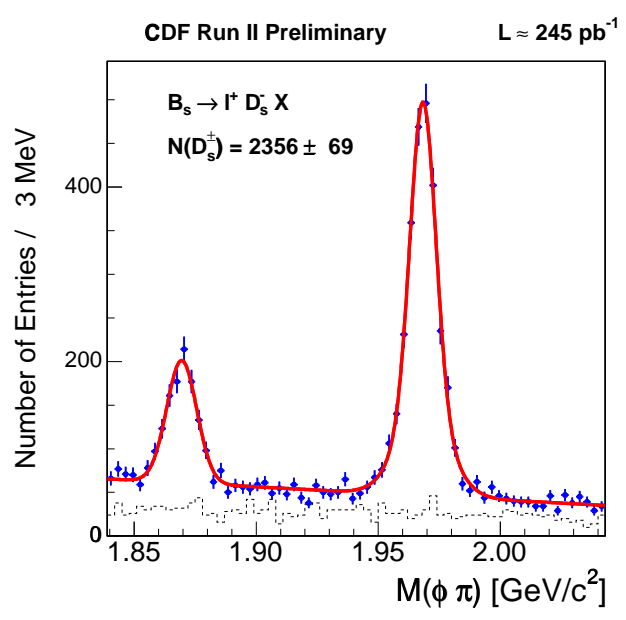

Figure 13. Semileptonic $B_{s}$ yields for CDF

necessary:

- Reconstruct an high statistic sample of $B_{s}^{0}$ decays.

- Determine the $B_{s}^{0}$ proper time with high precision.

- Identify the flavor of the $B_{s}^{0}$ at the production and decay time.

The first two requirements are somehow in competition. The large samples of semileptonic decays shown in figure 12 for the D0 experiments and in figure 13 for $\mathrm{CDF}$, have poor proper time resolution due to the uncertainty on $\beta \gamma$ factor for the neutrino loss. Fully reconstructed $B_{s}^{0}$ decays have a good proper time resolution but lower statistics (see figure 14). In table 1 the yields for the decay modes currently reconstructed are summarized. The other crucial ingredient is the flavor tagging to determine whether a $B_{s}^{0}$ or a $\bar{B}_{s}^{0}$ was produced. Several algorithms have been developed based on the study of the fragmentation tracks around the $B$ (Same Side Tag) or which identify the flavor of the other $B$ in the event (Opposite Side Tag). The effectiveness of a flavor tagger is usually expressed as $\epsilon D^{2}$, where $\epsilon$ is the tagger efficiency and $D=1-2 W$ is the dilution with $W$ 


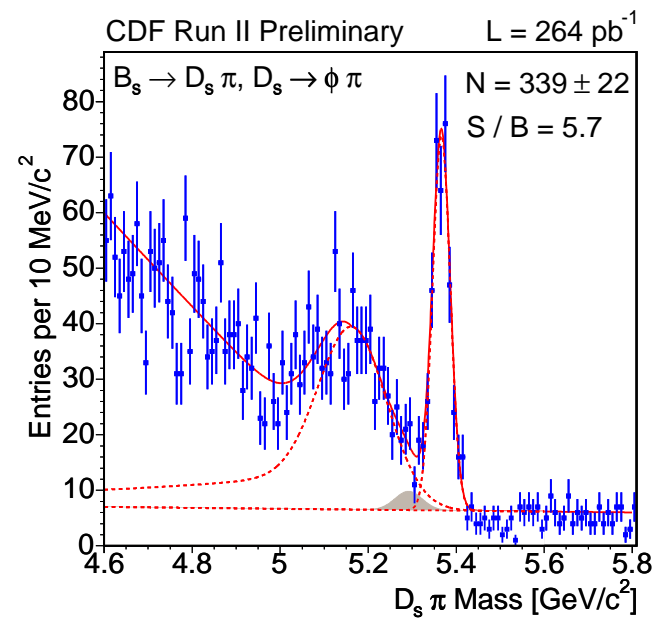

Figure 14. $B_{s} \rightarrow D_{s} \pi D_{s} \rightarrow \phi \pi$ yields as reconstructed at $\mathrm{CDF}$

Table 1. $B_{s}^{0}$ yields for CDF and, last row, for D0. $N^{o b s}$ is the observed number of events and $L$ is the integrated luminosity.

\begin{tabular}{l|c|c} 
Channel & $N^{o b s}$ & $L\left(\mathrm{pb}^{-1}\right)$ \\
\hline \hline $\begin{array}{l}B_{s}^{0} \rightarrow D_{s} \pi \\
\left(D_{s} \rightarrow \phi \pi\right)\end{array}$ & $339 \pm 22$ & 264 \\
\hline$B_{s}^{0} \rightarrow D_{s} \pi$ & $57 \pm 11$ & 124 \\
$\left(D_{s} \rightarrow 3 \pi\right)$ & & \\
\hline $\begin{array}{l}B_{s}^{0} \rightarrow D_{s} \pi \\
\left(D_{s} \rightarrow K^{*} K\right)\end{array}$ & $190 \pm 25$ & 240 \\
\hline $\begin{array}{l}B_{s}^{0} \rightarrow D_{s} 3 \pi \\
\left(D_{s} \rightarrow \phi \pi\right)\end{array}$ & $95 \pm 17$ & 264 \\
\hline$B_{s}^{0} \rightarrow l \nu D_{s} X$ & $2342 \pm 66$ & 245 \\
$\left(D_{s} \rightarrow \phi \pi\right)$ & & \\
\hline$B_{s}^{0} \rightarrow l \nu D_{s} X$ & $9481 \pm 253$ & 250 \\
$\left(D_{s} \rightarrow \phi \pi\right)$ & & \\
\hline \hline
\end{tabular}

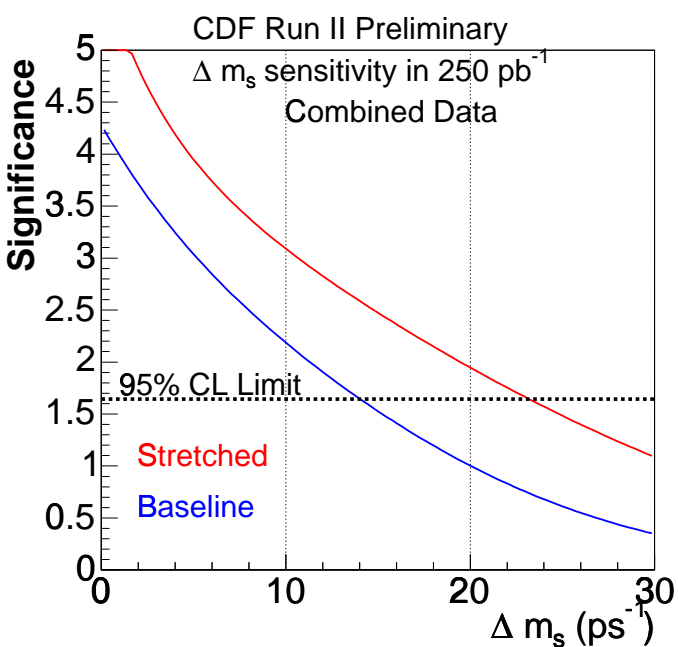

Figure 15. $B_{s}^{0}$ mixing sensitivity plotted as the significance as function of $\Delta m_{s}$ for two scenario: stretched and baseline

the wrong tagging probability. Both experiments have demonstrated their capability of tagging events by measuring the oscillation frequency in the $B_{d}^{0}$ system. When all tagging algorithms are combined CDF obtains $\epsilon D^{2}=1.820 \pm 0.114 \%$. D0 has similar values.

Neither CDF nor D0 have at the moment enough data to measure $\Delta m_{s}$. Both collaboration are working to set a limit. CDF has studied its sensitivity for different configurations which are close to what is observed in the data. The current dataset consists of 250 $\mathrm{pb}^{-1}$ of data. The predictions are calculated for two different scenario: $\epsilon D^{2}=1.6 \%$ and the proper time resolution, $\sigma_{t}=67 \mathrm{fs}$ called "baseline" and $\epsilon D^{2}=2.6 \%$ and $\sigma_{t}=47 \mathrm{fs}$ identified as "stretched". In figure 15 the significance is plotted as function of $\Delta m_{s}$ for the combined sample of semileptonic and hadronic decays. The $95 \%$ Confidence Level (CL) limit is $\Delta m_{s} \approx 14 \mathrm{ps}^{-1}$ for the baseline scenario and $\Delta m_{s} \approx 23 \mathrm{ps}^{-1}$ for the streched one. The same numbers can be used to evaluate the reaches. The sensitivity for 


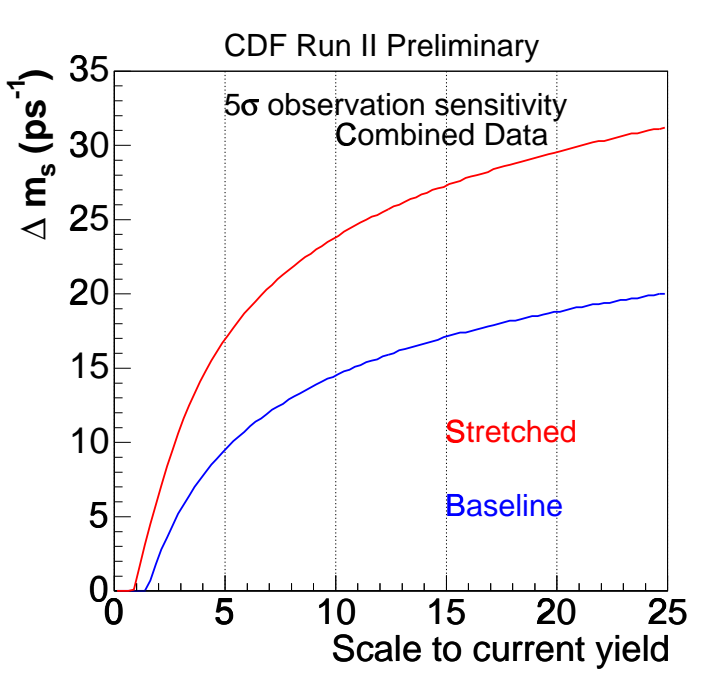

Figure 16. $B_{s}^{0}$ mixing sensitivity for $5 \sigma$ observation as function of the scale factor to the current yield

$5 \sigma$ observation of a given $\Delta m_{s}$ is shown in figure 16 as function of a scale factor respect to the current yield (which does not just multiply the current yield, because of the detector and trigger configurations which can change as the luminosity increase) of combined hadronic and semileptonic decay modes. In order to measure $\Delta m_{s}=19$ $\mathrm{ps}^{-1} \mathrm{CDF}$ needs 6 times more data respect to what has now and 10 times to reach $\Delta m_{s}=24 \mathrm{ps}^{-1}$.

\section{Conclusions}

This review presents a snapshot of the Jet and Heavy Flavor physics from the Tevatron experiments in summer 2004. The jet data have produced several interesting results. The inclusive cross section and the dijets cross section have been studied to search for new physics and to verify the pQCD with high precision at high momentum. The measurements done at low momentum allow to study the non perturbative process like soft gluon contributions and fragmentation. The physics results achieved with $c$ and $b$ hadron are already of primary importance to test the Standard Model and the new data that both collaborations are analyzing will be useful to better constrain it. The $B_{s}^{0}$ physics provide a unique window on $B$ physics and it is accessible only at the Tevatron. The measurement of $B_{s}^{0}$ oscillation frequency is one of the most important to constrain the heavy flavor sector of Standard Model together with the $B_{s}^{0}$ lifetime difference. A discrepancy with the model would mean new physics. Beside that, the Tevatron can also contribute to other $B$ measurements competing with the $B$-factories since the number of events collected are becoming really huge.

\section{References}

1. F. Chlebana, these proceedings.

2. M. Cacciari and P. Nason JHEP, 09006 (2003).

3. P. De Jong, these proceedings.

4. F. Gabbiani, A. I. Onishchenko and A. A. Petrov, arXiv:hep-ph/0407004.

5. P. Gutierrez, these proceedings.

6. M. Rescigno, these proceedings.

7. G. Punzi, these proceedings.

8. A. Cerri, these proceedings.

9. C. Chang and Y. Chen, Phys. Rev. D49 (1994) 3399.

10. D.Ebert, R.N. Faustov, V.O. Galkin (2004) hep-ph,0401237.

11. D.Ebert et al, Phys. Rev. D68 (2003) 094020.

12. S. Towers, these proceedings.

13. ALEPH Collab., Phys. Lett B425, 215 (1998).

14. E.J.Eichten, C.T. Hill, C. Quigg, Phys. Rev. Lett. 714116 (1993).

15. N. Isgur, Phys. Rev D57, 4041 (1998).

16. D. Ebert, V.O. Galkin, R.N. Faustov, Phys. Rev. 575663 (1998).

17. A.H.Orsland, H. Hogaasen Eur. Phys $J$ C9 503 (1999). 